\title{
Improving post-operative communication between primary and secondary care: the wound closure information card
}

\author{
Isabella Dash ${ }^{1, a}$ and Greg T. Pickering ${ }^{2, a}$ \\ ${ }^{1}$ ST7 Registrar in General Surgery, Royal United Hospital Bath NHS Foundation Trust, Bath, UK \\ ${ }^{2}$ ST5 Registrar Trauma \& Orthopaedic Surgery, Royal United Hospital Bath NHS Foundation Trust, Bath, UK
}

\begin{abstract}
Aim: To assess and improve the quality of Secondary to Primary Care communication on discharge with a focus on post-surgical wound care. Background: Hospital discharge summaries are the principle means of relaying accurate information back to primary care healthcare providers regarding a patient's hospital attendance and any ongoing care that is required. The quality of these summaries can be quiet varied both nationally and local to our Trust. Subsequently the Surgical Directorate were seeing an increased level of additional emergency communication from Primary Care providers especially in relation to postoperative wound care. Methods: A survey was distributed to local Primary Care practitioners to assess satisfaction with the General Surgical Department wound care information located on the discharge summary. Using these results, a wound closure information document was developed and distributed to general practice surgeries, and a patient-held 'wound care' card was piloted for two months. The survey was then repeated to determine the success of the intervention. Findings: Post discharge communication was on the whole felt to be of poor quality and lacked a large amount of essential and desirable information. There was a particular absence of relevant information regarding surgical wound closure techniques utilised and their ongoing management. Many Primary Care practitioners acknowledge that their knowledge on this subject can be low. A Trust specific information leaflet combined with a dedicated patient held discharge information card can solve a number of these issues improving Primary and Secondary Care satisfaction and reducing the use of emergency resources and appointments.
\end{abstract}

Key words: patient discharge; patient information; primary care and secondary care communication; quality improvement; wound care

Received 23 February 2015; revised 3 January 2016; accepted 28 January 2016;

first published online 16 June 2016

\section{Introduction}

With over 15 million hospital admissions per year in the UK (Health \& Social Care Information Centre, 2014), hospital discharge summaries are

Correspondence to: Greg T. Pickering, Department of Trauma \& Orthopaedics, Royal United Hospital Bath NHS Foundation Trust, Combe Park, Bath BA1 3NG, UK. Email: greg.pickering@nhs.net

${ }^{a}$ Isabella Dash is an ST7 Registrar in General Surgery at the Royal United Hospital Bath NHS Foundation Trust. Greg Pickering is an ST5 Registrar in Trauma \& Orthopaedics at Royal United Hospital Bath NHS Foundation Trust. the principle means of relaying accurate information back to primary care healthcare providers regarding a patient's hospital attendance, their received treatment and any ongoing care that is required. In the UK, the primary care healthcare providers consist of general practitioners (GP), practice nurses and district nurses, who all work within a 'GP surgery', and will be able to access discharge summaries from hospitals (secondary care providers), as well as providing ongoing care for patients in the community following hospital discharge. Criticisms exist of delays between

\footnotetext{
(C) Cambridge University Press 2016
} 
patients' actual date of discharge and the date that GPs receive appropriate information. In one paper the maximum time between discharge and receipt was 27 days due to dictation and typing delays alone (Farquhar et al., 2005). With the advent of electronic discharge summaries this should be less of a problem; however, such systems are still in their relative infancy and the risk of delay, failure to correctly send and reliably receive the relevant information exists. Ideally, the patient is given a physical copy of their discharge summary or care plan to take home with them whilst an electronic copy is emailed to their GP within $24 \mathrm{~h}$ (Department of Health, 2003). The Clinical Data Standards Assurance Programme was set up in August 2010 to deliver a national and clinically assured electronic discharge summary that the acute hospital team would send out to the GP within $24 \mathrm{~h}$ of discharge (Health \& Social Care Information Centre, 2014). Minimum standards of information which should be included are as follows (Katikireddi and Cloud, 2008): reason for admission, other significant events or illnesses during admission, operations or therapeutic procedures undergone, medication changes, and a clear ongoing primary and secondary care plan stating further investigations, interventions and follow-up appointments as appropriate.

The content of discharge information is often poor (Luker et al., 2000; Harel et al., 2012) as the summaries are often written by the most junior member of the hospital team. They are often reliant on information in the medical records and not always on a direct, personal knowledge of the patient being discharged. The relatively low priority that formal discharge paperwork often has compared with other acute clinical care tasks drawing on a junior hospital doctor's time and attention makes their completion more often than not retrospective in nature compounding the delay and poor content quality. Delayed discharge summaries have been found to be associated with an increased rate of hospital readmissions, especially in the elderly population (Li et al., 2013) and educational strategies (with various effects) have been implemented in various hospitals worldwide to improve the content of discharge summaries, highlighting that this is a global problem (O'Leary et al., 2009; Key-Solle et al., 2010).

Our trust serves a catchment population of $\sim 500000$. Outside of the city centre the local area is predominantly rural with a number of small towns and villages. Within the trust it was noted by those doctors 'on call' for general surgery that they would frequently receive telephone calls from frustrated primary care providers (GPs or practice nurses) that either no discharge summary had been received or that those that had been sent were missing key information, primarily with regards to post-operative care and ongoing wound closure management. Patients would frequently present to their practice post-operatively for routine wound review. Unless formally documented on the discharge summaries, primary care providers would regularly be unaware of when the sutures or clips should be removed, if even required at all.

We set out to identify the knowledge levels on current wound closure techniques and their management amongst local GP practices. Once established, we aimed to instigate an intervention to both improve knowledge on this important and relevant topic as well as facilitate secondary to primary care communication. We defined wound closure as closure of any incision that had been made in the skin for the purpose of a general surgery operation, which had been closed primarily, with either sutures or clips.

\section{Methods}

We emailed an anonymous SurveyMonkey ${ }^{\mathrm{TM}}$ survey out to all surrounding 27 GP practices via our hospital's Education Department, assessing the staff at each practice's knowledge regarding current methods of wound closure being utilised within our trust. This could be filled in by a GP or a practice nurse as both are key members of the primary care team. Results were then reviewed for perceived confidence and knowledge on wound care, as well as opinions regarding the current system of conveying such information post surgery and patient discharge.

Subsequently, a trust-specific information leaflet on the topic 'wound closure methods' (Figure 1), currently being employed by the hospital, along with their required follow-up and management was designed and distributed to all of the above GP practices.

In an attempt to eliminate the potential time delay in transference of this relevant wound care information and in doing so both meet the UK Department of Health clinical guidelines (2003) on best practice and maximise patient healthcare 


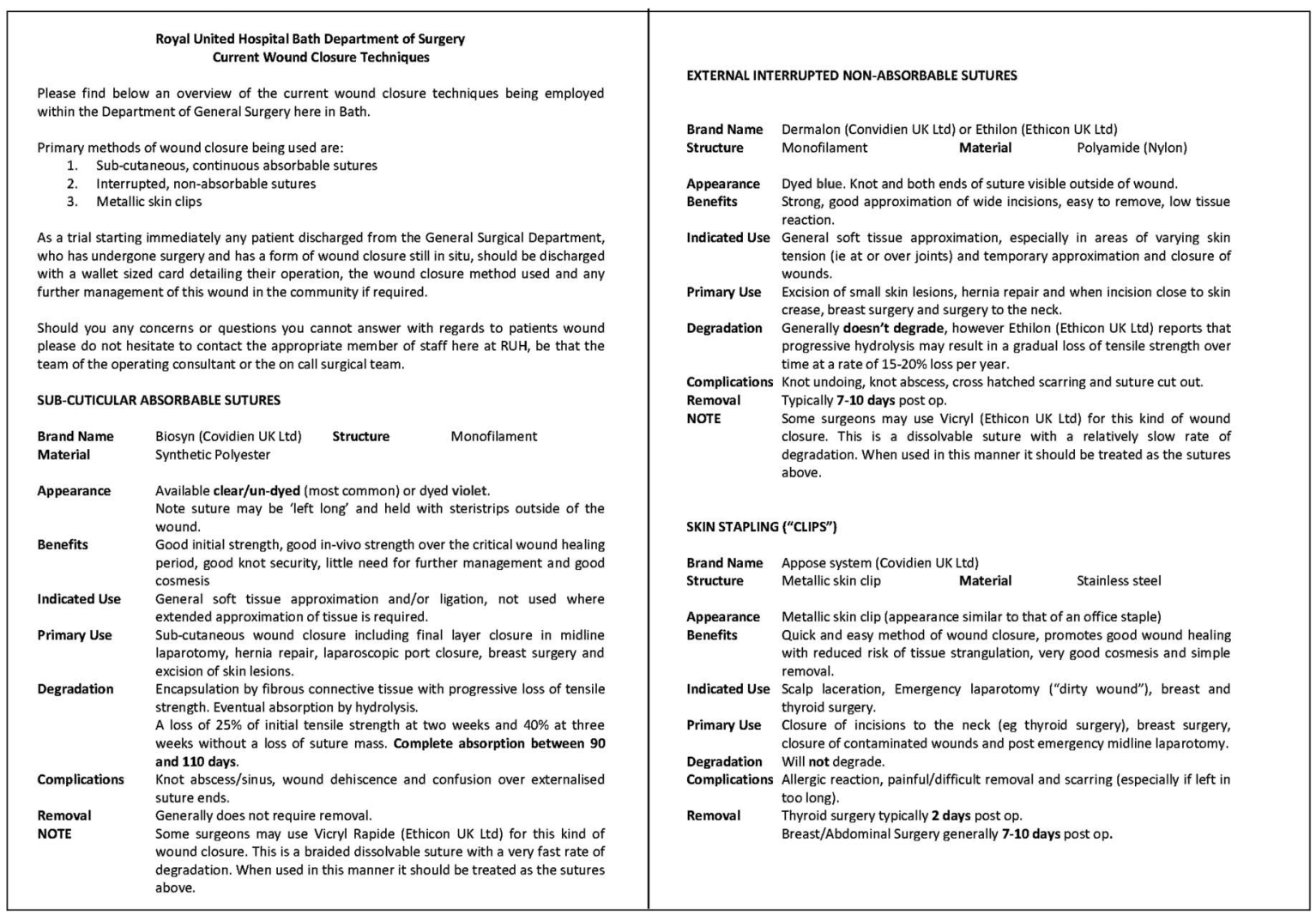

Figure 1 Trust-specific suture information sheet distributed to all surrounding primary care practices

ownership empowerment, a wallet-sized personalised information card was designed for the patient to take home. This detailed the name and date of the operation that the patient had undergone, the type of wound closure employed and if requiring removal, the date. This was then completed and given to all general surgical patients on discharge to keep on their person and piloted over a two-month period.

The GP practices were re-surveyed two months later, asking whether any changes had been noted and perceived knowledge levels following wound closure information sheet distribution.

\section{Results}

In total, $22(81 \%)$ of 27 GP practices surveyed replied, with a mixture of responses coming from GPs and practice nurses (Figure 2). Of these, only a third of respondents $(86 \%$ of whom were practice nurses) stated that they were satisfied with the information regarding surgical wound closure currently supplied on the discharge summaries they received. Another third of respondents were dissatisfied, $66 \%$ of these were GPs, with the final third feeling indifferent (Figure 3).

When the relevant information was present on written/electronic discharge paperwork, over half of those surveyed (70\% GPs) found the information difficult or very difficult to find and understand (Figure 4).

The majority of primary care providers had patients present to them with post-operative wound queries or problems at least fortnightly.

In total, $70 \%$ of practices felt that an up-to-date trust-specific wound closure information would be extremely useful, and that a readily distributed personalised patient-held card could be both practical and efficient. 


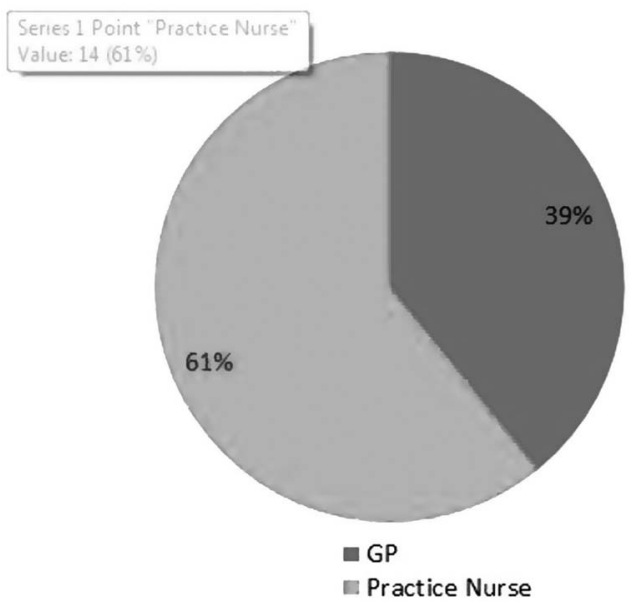

Figure 2 Proportion of responses from GPs and practice nurses

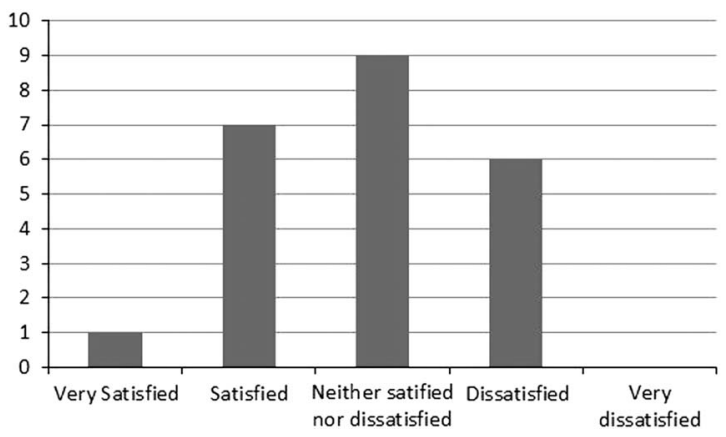

Figure 3 Satisfaction with general surgery discharge summaries

Following distribution of the trust wound closure leaflet and a two-month trial of the patientheld information cards, a quarter of practices had encountered and utilised the patient-specific card, whilst the majority (16 of 19 replies) felt that wound care information availability and their own personal knowledge on the topic had improved.

\section{Discussion}

We found that within our surrounding primary care providers, there was general dissatisfaction with discharge summaries as a whole and within that, information concerning post-operative wound care in particular. This is similar to what has been

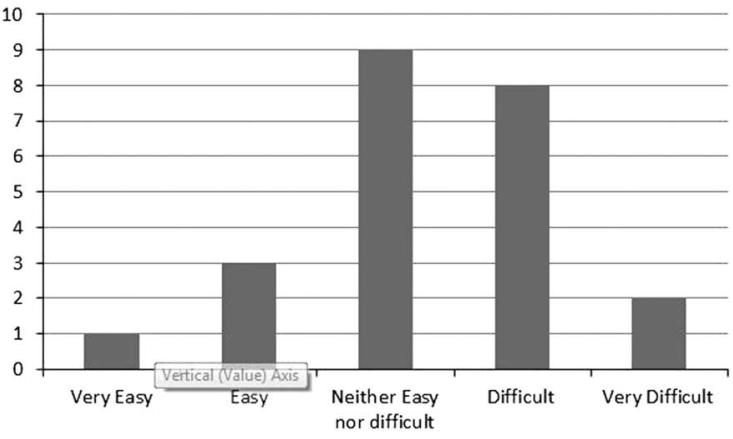

Figure 4 Ease at which to find skin closure information within discharge summary

found in the literature, a recent systematic review showed that poorly written communication between hospital specialists and primary care trusts can have many negative effects (Vermeir et al., 2015). Through the means of a targeted information sheet and patient-specific discharge cards, there was an improved sense of knowledge on the topic and a decreased need for the primary care team to ring the on-call surgical team for advice (noted both in the survey and anecdotally amongst surgeons). Such interventions also eliminate the time delay that may occur with routine discharge summaries. We found a positive response on re-surveying the practices, in that $60 \%$ of those responding felt that the information available to them on discharge specifically regarding wound closure had improved considerably.

In addition, the production and distribution of trust-specific information on the topic of surgical closure both reinforced the message that was trying to be delivered, as well as providing a simple reference document to both interpret the information provided on the discharge cards as well as offer further information in the event of a problem or ongoing uncertainty concerning a patient's post-operative wound care in the community.

Giving the patient the responsibility of keeping the card had the additional unanticipated benefit of allowing the patient to show any healthcare provider, whether an out of hours GP in the area or another hospital team if required, both their operation and which team had provided the care. Aside from the communication benefits, the patients themselves occasionally reported an improved sense of empowerment on discharge in knowing what

Primary Health Care Research \& Development 2017; 18: 92-96 
their operation was and how it would continue to be managed in the short term post discharge, when questioned informally in the outpatient clinic.

No formal cost analysis of this intervention has been performed due to the currently limited size of the trial performed and the associated costs being low. The suture information sheets were compiled by the study investigators from information available in the public domain, and following approval from the trust's communications team were distributed electronically. The initial trial of patient-held information cards was funded from the local departmental research fund. Such cards cost less than $£ 0.01$ (GB Pounds) per unit to produce. It is anticipated that these costs could be factored into the total cost of operative care per patient.

Following its success, the wound care card has now been adapted and incorporated into a trust-wide leaflet entitled 'Looking after your surgical wound', which is given to all surgical patients on discharge. This provides both specific and general information with regards to post-operative wound care, that is, minimising infection, as wellas what the wound closure method utilised was.

\section{Conclusion}

Good communication between secondary and primary care is key in order to ensure patient's health needs are met. This simple intervention facilitated better communication by the distribution of prompt and specific information via the patient directly. This should help save time and money through decreasing unnecessary telephone consultations and emergency admissions, and subsequently reducing the workload for both those in primary and in secondary care in the future.

\section{Acknowledgements}

We would like to acknowledge the support of Mr Mike Williamson as project supervisor and Louise Corrigan for assistance with design and construction of the patient information leaflet.

\section{Financial Support}

This research received no specific grant from any funding agency, commercial or not-for-profit sectors.

\section{Conflicts of Interest}

None.

\section{References}

Department of Health. 2003: Department of Health clinical guidelines: discharge from hospital, pathway, process and planning. London: Department of Health.

Farquhar, M.C., Barclay, S.I., Earl, H., Grande, G.E., Emery, J. and Crawford, R.A. 2005: Barriers to effective communication across the primary/secondary interface: examples from the ovarian cancer patient journey (a qualitative study). European Journal of Cancer Care 14, 359-66.

Harel, Z., Wald, R., Perl, J., Schwartz, D. and Bell, C.M. 2012: Evaluation of deficiencies in current discharge summaries for dialysis patients in Canada. Journal of Multidisciplinary Healthcare 5, 77-84.

Health \& Social Care Information Centre. 2014: Electronic 24-hour discharge summary implementation. Retrieved 21 December 2014 from http://systems.hscic.gov.uk/ clinrecords/24hour/index_html.

Katikireddi, S.V. and Cloud, G.C. 2008: Planning a patient's discharge from hospital. BMJ 337(a), 2694-97.

Key-Solle, M., Paulk, E., Bradford, K., Cockrell Skinner, A., Lewis, M.C. and Shomaker, K. 2010: Improving the quality of discharge communication with an educational intervention. Paediatrics 126, 734-39.

Li, J.Y., Yong, T.Y., Hakendorf, P., Ben-Tovim, D. and Thompson, C.H. 2013: Timeliness in discharge summary dissemination is associated with patients' clinical outcomes. Journal of Evaluation in Clinical Practice 19, 76-79.

Luker, K., Beaver, K., Austin, L. and Leinster, S.J. 2000: An evaluation of information cards as a means of improving communication between hospital and primary care for women with breast cancer. Journal of Advanced Nursing 31, 1174-82.

O'Leary, K.J., Liebovitz, D.M., Feinglass, J., Liss, D.T., Evans, D.B., Kulkarni, N., Lander, M.P. and Baker, D.W. 2009: Creating a better discharge summary: improvement in quality and timeliness using an electronic discharge summary. Journal of Hospital Medicine 4, 219-225.

Vermeir, P., Vandijk, D., Degroote, S., Peleman, R., Verhaeghe, R., Mortier, E., Hallaert, G., Ven Daele, S., Buylaert, W. and Vogelaers, D. 2015: Communication in healthcare: a narrative review of the literature and practical recommendations. The International Journal of Clinical Practice 69, 1257-67. 